\title{
Angular streaking in strong field ionization of chiral molecules
}

\author{
K. Fehre $\odot,{ }^{1, *}$ S. Eckart, ${ }^{1}$ M. Kunitski, ${ }^{1}$ C. Janke, ${ }^{1}$ D. Trabert,${ }^{1}$ J. Rist,${ }^{1}$ M. Weller,${ }^{1}$ A. Hartung, ${ }^{1}$ M. Pitzer, ${ }^{2}$ \\ L. Ph. H. Schmidt, ${ }^{1}$ T. Jahnke, ${ }^{1}$ R. Dörner, ${ }^{1, \dagger}$ and M. S. Schöffler ${ }^{1, \neq}$ \\ ${ }^{1}$ Institut für Kernphysik, J. W. Goethe-Universität Frankfurt, Max-von-Laue-Str. 1, 60438 Frankfurt am Main, Germany \\ ${ }^{2}$ Department of Chemical Physics, Weizmann Institute of Science, P.O. Box 26, 76100 Rehovot, Israel
}

(Received 13 June 2019; published 23 October 2019)

\begin{abstract}
We report on a chiral observable upon ionization of a chiral molecule (methyloxirane) by a strong elliptically polarized laser field: a rotation of the photoelectron momentum distribution, which is enantiosensitive and forward/backward asymmetric. We explain this forward/backward asymmetric rotation of the count maxima to be equivalent to a dependence of the photoelectron circular dichroism on the electron emission angle in the plane of polarization.
\end{abstract}

DOI: 10.1103/PhysRevResearch.1.033045

\section{INTRODUCTION}

The interaction between chiral molecules and chiral light leads to qualitatively novel symmetry properties in the photoelectron distribution, which do not exist for achiral species. Photoelectron circular dichroism (PECD) is a prominent example which is an enantiomer-dependent asymmetry of the electron emission probability with respect to the light propagation direction. PECD has proven its potential as a sensitive analysis method in chemistry and pharmacy [1-3]. PECD is also a versatile tool for studying photoionization dynamics [4]. PECD mostly originates from the interaction of the photoelectron with the chiral potential of the ion (see Ref. [5] for the role of intermediate states). This makes it particularly intriguing to extend our understanding of PECD towards the strong field (tunneling) regime where electron wave packets are commonly modeled to be driven by the strong light field after they exit the classical forbidden region (tunnel). Here the laser pulse imparts a vectorial momentum onto the electron, which is given by the vector potential at the birth time of the photoelectron wave packet. It is not at all obvious what the role of a chiral potential will be in this case.

In this paper, we study the interplay between angular streaking and the chiral potential. We show that a characteristic change of the so-called "attoclock angle" depends on the enantiomer and elucidate how this is connected to PECD.

A benefit of using circularly polarized light in the tunneling regime is that the momentum imparted by the laser field on the emitted particle maps the time of ionization within the laser pulse onto the particle's emission angle. This establishes an

\footnotetext{
*fehre@atom.uni-frankfurt.de

†doerner@atom.uni-frankfurt.de

‡schoeffler@atom.uni-frankfurt.de
}

Published by the American Physical Society under the terms of the Creative Commons Attribution 4.0 International license. Further distribution of this work must maintain attribution to the author(s) and the published article's title, journal citation, and DOI. ultrafast clock where the rotating electric field vector acts as clockwork and the electron momentum vector serves as the hand of the clock. This concept of angular streaking [6,7] has been used to time single [8] and double tunnel ionization [9] and to study time correlations between ionization and dissociation [10]. The interpretation of the measured emission angle requires a reference, i.e., a zero of the angle. For a molecular process, the molecular axis can serve this purpose. For double ionization one of the two emitted electrons can be taken as a reference. For single ionization by elliptically polarized light, the major axis of the polarization ellipse is a natural reference. The emission angle $\varphi$ in the polarization plane encodes a combination of the birth time and the initial momentum of the wave packet [11] as well as its interaction with the ionic potential. It is this coordinate in which we search for a chiral signature.

\section{EXPERIMENTAL METHOD}

A symmetric cold target recoil ion momentum spectroscopy [12] spectrometer was built consisting of two identical arms $(21 \mathrm{~cm}$ acceleration length and $E=119 \mathrm{~V} / \mathrm{cm}$ electric field). Both sides of the spectrometer were equipped with a detector [Photonis multichannel plate (MCP); open area ratio (OAR) specified $60 \%$, slightly used, electron detector]/Hamamatsu MCP (OAR specified 90\%, recoil detector [13]), and a second MCP for further amplification followed by a hexagonal delay-line anode [14] for position decoding. The main chamber was baked for 1 wk at $90{ }^{\circ} \mathrm{C}$, resulting in a residual gas pressure without a gas jet of $1 \times 10^{-10}$ mbar. The ionization of the methyloxirane molecules was induced by focusing a short, intense, laser pulse $(f=60 \mathrm{~mm}, 8 \mathrm{~mm}$ beam diameter, $40 \mathrm{fs}$, central wavelength $800 \mathrm{~nm}, 0.3 \mathrm{~W}$ ), generated by a Ti:sapphire regenerative amplifier (KMLabs, Wyvern 500 ), resulting in a peak intensity of $1.3 \times 10^{14} \mathrm{~W} / \mathrm{cm}^{2}$ onto the supersonic gas jet. The laser intensity was obtained by setting our quarter-wave plates to produce circular polarized light using the same pulse energy. There we measured the mean transversal electron momentum $p_{e t \text {, mean }}=\sqrt{p_{e y}^{2}+p_{e z}^{2}}$, and assumed that this corresponds to the vector potential 

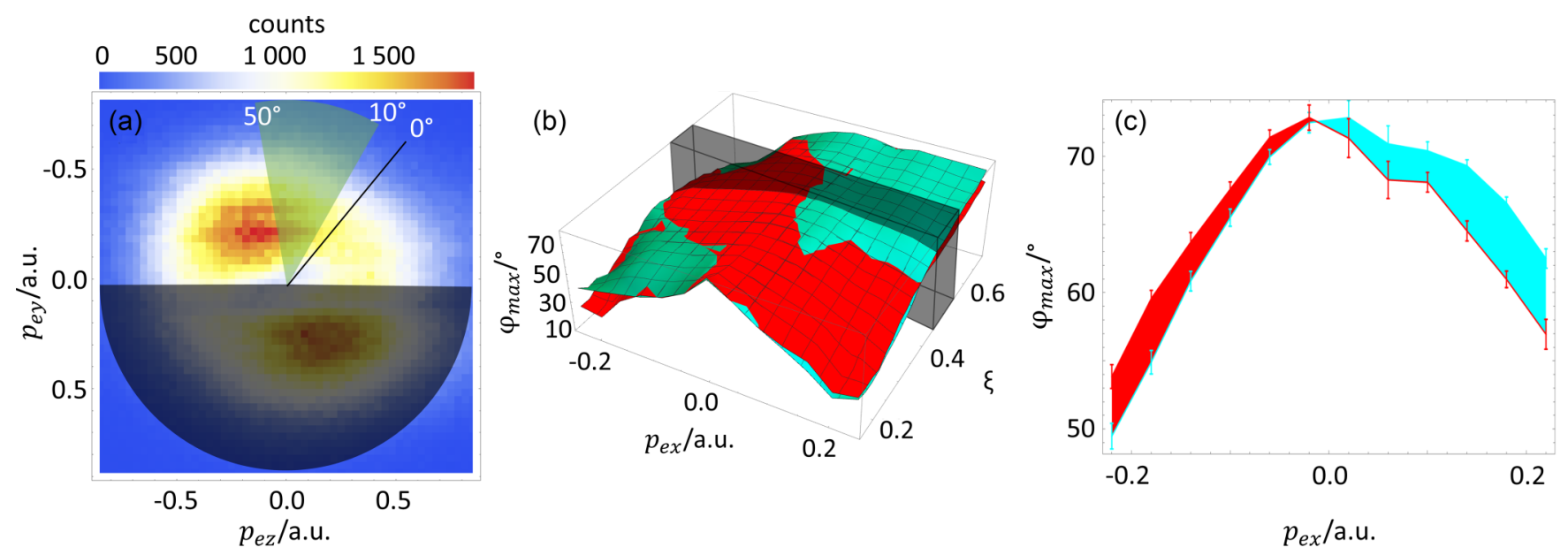

FIG. 1. Single ionization of methyloxirane by elliptically polarized laser pulse with a field ratio of $\frac{E 1}{E 2}=0.7$. (a) shows a projection of the electron momentum distribution onto the plane of polarization $(z, y)$ in Cartesian coordinates. The gray shaded area $\left(p_{e y}>0\right)$ is excluded from the Gaussian fit presented in (b). Attoclock angle of the count maximum $\varphi_{\max }\left(p_{e x}, \xi\right)$ for a given $p_{e x}$ and $\xi=\sqrt{p_{e y}^{2}+s^{2} p_{e z}^{2}}$, extracted as shift of the Gaussian fit for each cut in $p_{e x}$ and $\xi$. The parameter $s$ modifies the transformation into elliptical coordinates and was chosen such that the mean value of $\xi$ is independent of $\varphi$. Data for the $R$ enantiomer is displayed in cyan, and for the $S$ enantiomer in red. The cut in $\xi(0.48<\xi<0.58$, gray shaded area) is displayed in (c). The error bars reflect the standard error of the shift in the Gaussian fit. The green shaded area in (a) indicates the selection in $\varphi$, used in Fig. 3.

$E_{o} / \omega_{L}$ [15]. From this, the peak intensity for $\varepsilon=0.7$ was calculated. With the ionization potential of $10.25 \mathrm{eV}$ [16] (methyloxirane), this results in a Keldysh parameter of $\gamma=$ 0.8 . Switching the helicity of the light with a motorized $\lambda / 4$-wave plate every $3 \mathrm{~min}$ ensured identical experimental conditions for left- and right-handed elliptically polarized light. The jet was produced by expanding methyloxirane with its vapor pressure at room temperature ( $\sim 588 \mathrm{mbar})$ through a nozzle of $30 \mu \mathrm{m}$ diameter into vacuum. Left- and right-handed circular polarization were set via the minimum count rate. The ellipticity for LEP/REP (left-/right-handed elliptical polarization) and thus the relation between $E 1$, the electric field strength of the minor axis, and $E 2$, the electric field strength of the major axis, was calculated from the position of the wave plates. The photoelectrons were measured in coincidence with the singly charged parent ions as the fragmentation channel strongly influences the PECD signal in the strong field regime [17].

\section{ENANTIOSENSITIVE ROTATION OF THE PHOTOELECTRON MOMENTUM DISTRIBUTION}

We singly ionize enantiopure methyloxirane (SigmaAldrich) by a 40 -fs laser pulse with a central wavelength of $800 \mathrm{~nm}$ and a peak intensity of $1.3 \times 10^{14} \mathrm{~W} / \mathrm{cm}^{2}$. By measuring the photoelectrons in coincidence with the singly charged parent ion we select mainly ionization from the highest occupied molecular orbital [2]. Figure 1(a) shows the electron momentum distribution in the polarization plane $\left(p_{e y}, p_{e z}\right)$. The two maxima in the distribution correspond to ionization at the opposite peaks of the laser electric field.

For the following we use elliptical coordinates where $\xi=$ $\sqrt{p_{e y}^{2}+s^{2} p_{e z}^{2}}$ is the radial momentum in the polarization plane and $p_{e x}$ is the momentum component along the light propagation. The coordinates $p_{e y}$ and $p_{e z}$ were rotated such that the direction of the major axis of the laser polarization lies in the $p_{e z}$ direction. The value $s=0.82$ was determined so that the mean value in $\xi$ was independent of $\varphi_{1}=$ $a \tan 2\left(p_{e y}, p_{e z}\right)+\varphi_{1,0}$. We examine the angle of the distribution maxima $\varphi_{\max }\left(p_{e x}, \xi\right)$. In order to quantify it, we perform Gaussian fits for different pairs of $p_{e x}$ and $\xi$ (i.e., along ellipses in the $p_{e y}-p_{e z}$ plane in Cartesian coordinates) to obtain $\varphi_{\max }$ for the count maximum. Figure 1(b) displays $\varphi_{\max }\left(p_{e x}, \xi\right)$ for elliptical polarized light with counterclockwise rotating electrical field (LEP) and the two enantiomers. The first distinctive feature, which is common to both enantiomers, is the strong dependence of $\varphi_{\max }$ on $p_{e x}$. We observe that the angle varies about $40^{\circ}$ over the displayed range of $p_{e x}$. This striking effect is universal to strong field ionization. We have observed this effect in other experiments on achiral molecules as well as on atoms under different laser field intensities. This rotation as a function of $p_{e x}$ is a direct consequence of the ionic Coulomb potential onto the escaping electron. The final momentum component $p_{e x}$ is not created by the laser field but results from the initial momentum at the tunnel exit as no laser field component is present in the $x$ direction. The electrons with large $p_{e x}$ escape the vicinity of the ion more rapidly and thus experience less interaction with the Coulomb potential. Therefore, with increasing $\left|p_{e x}\right|$, the direction of the final electron momentum in $\varphi$ approaches the direction of the laser's vector potential, while for $p_{e x}=0$ the influence of the Coulomb field on the trajectory maximizes and $\varphi_{\max }$ differs from the angle predicted by the vector potential.

More intriguing is the more subtle effect, that the position of $\varphi_{\max }$ depends on the enantiomer, on $\xi$, and on the sign of $p_{e x}$ as shown in Fig. 1(b) where the red and green surfaces show $\varphi_{\max }$ for the $S$ and $R$ enantiomers, respectively. For each enantiomer $\varphi_{\max }$ is forward/backward asymmetric and displays a strong dependence on $\xi$. Moreover, the enantiomers show to some extent the mirror-symmetric behavior in $p_{e x}$. 


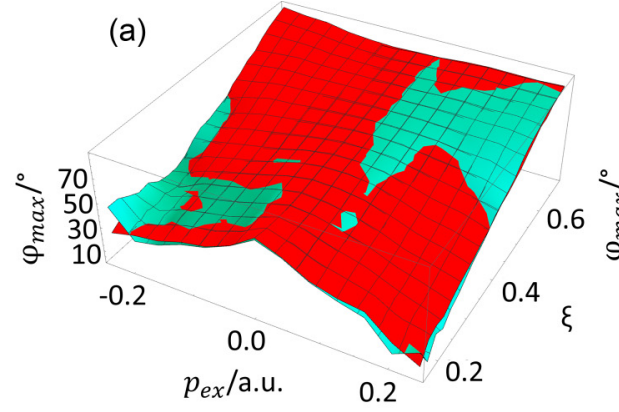

(d)

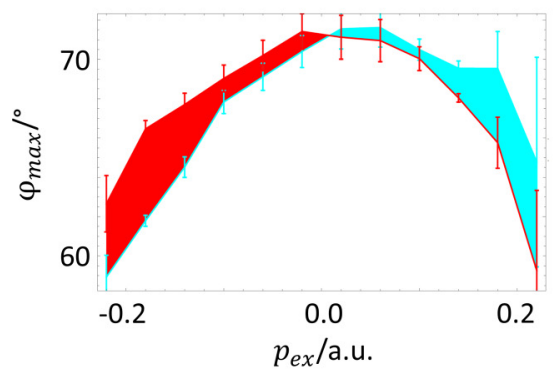

(b)

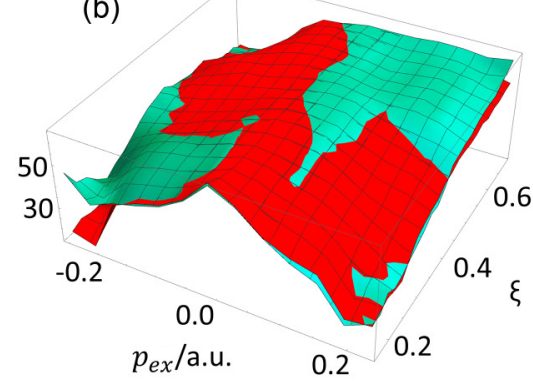

(e)

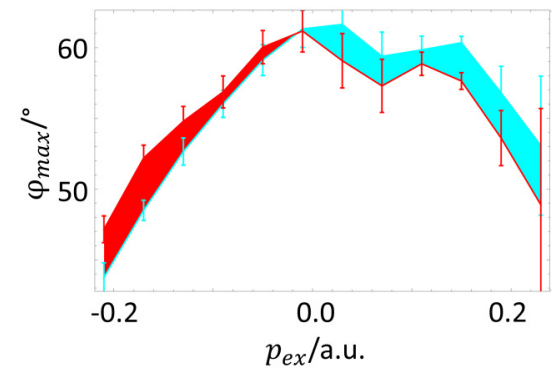

(c)

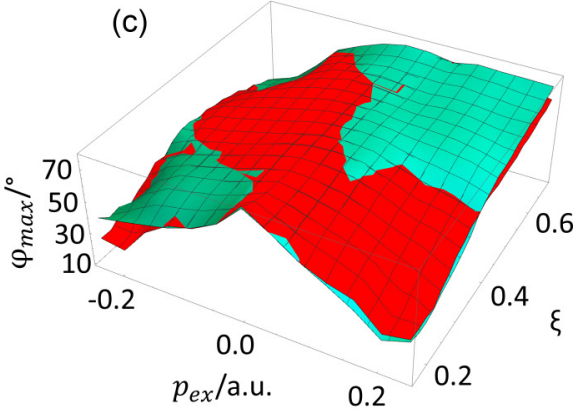

(f)

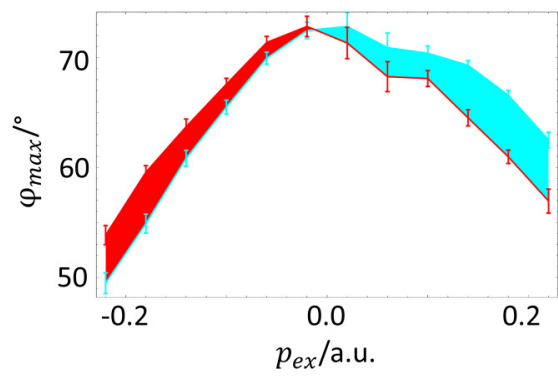

FIG. 2. Discussion of the Gaussian fits for different coordinate systems. Attoclock angles for given $p_{e x}$ and $\xi$ are extracted from the three-dimensional electron distribution of LEP for a field ratio of $\frac{E 1}{E 2}=0.7$. Data for the $R$ enantiomer is displayed in cyan, and for the $S$ enantiomer in red. (a) $\varphi_{\max }\left(p_{e x}, \xi\right)$, fitted along circles as a function of $p_{e x}$ and $\xi=\sqrt{p_{e y}^{2}+p_{e z}^{2}}$. (b) $\varphi_{\max }\left(p_{e x}, \xi\right)$, fitted along ellipses as indicated in variant 1. (c) $\varphi_{\max }\left(p_{e x}, \xi\right)$, fitted along ellipses as indicated in variant 2. (d)-(f) display cuts in $\xi(0.48<\xi<0.58)$ from (a)-(c), respectively. The error bars reflect the standard error of the mean of the Gaussian fit.

Thus, for elliptically polarized light the angular position of the electron momentum distribution maxima represents a strong chiral signal.

\section{DETAILS FOR THE COORDINATE TRANSFORMATION}

First, we would like to state that there are fundamental problems in choosing the right coordinate system. While the Coulomb interaction as a central force is spherically symmetric, the elliptically polarized light follows an elliptical symmetry and PECD is described as forward/backward asymmetry in the light propagation direction. Thus, there is no coordinate system that respects all symmetries. Based on the description of PECD, we limit our further considerations to cylindrical coordinates and elliptical coordinates with the direction of light propagation as the third dimension.

The main contribution to the final electron momentum originates from the negative vector potential of the electric laser field, which, after the ionization process, accelerates the electron even if it is far away from the remaining ion. For elliptically polarized light, the electron distribution is thus centered around the ellipse, which describes the negative vector potential. In order to observe shifts of the count maxima on this ellipse, each cut in $p_{e x}$ must therefore be subdivided not onto circles but on elliptical paths. Introducing the value $s=0.82$, we modify the usual transformation from $p_{e y}$ and $p_{e z}$ (the final electron momenta in the plane of polarization) to $\varphi_{i}$ and $\xi_{i}$, examining two possibilities:
Variant 1: The radius is stretched depending on $\varphi$, and the angle in the polarization plane is preserved.

$$
\begin{gathered}
\varphi_{1}=a \tan 2\left(p_{e y}, p_{e z}\right)+\varphi_{1,0}, \\
\xi_{1}=\sqrt{p_{e y}^{2}+p_{e z}^{2}} \sqrt{\sin \left(\varphi_{1}\right)^{2}+s^{2} \cos \left(\varphi_{1}\right)^{2}} .
\end{gathered}
$$

Variant 2: The main axes of the ellipse are stretched. Here the angle is not preserved.

$$
\begin{gathered}
\varphi_{2}=a \tan 2\left(p_{e y}, s p_{e z}\right)+\varphi_{2,0}, \\
\xi_{2}=\sqrt{p_{e y}^{2}+s^{2} p_{e z}^{2}} .
\end{gathered}
$$

$\varphi_{1,0}=40^{\circ}$ was chosen such that the twofold $\mathrm{PECD}_{\text {lin }}$ pattern is nicely displayed in Fig. 4.

The comparison between the Gaussian fits in cylinder coordinates and the two described variants for the elliptic coordinates shows, however, that the properties discussed in the main text do not depend qualitatively on the choice of the coordinates (see Fig. 2). These are the forward/backward and enantiosensitive asymmetric difference of the rotation of the count maxima and the sign change at about $\xi=0.4$ a.u.,

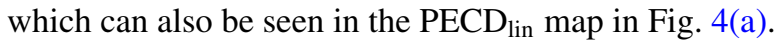

It should be noted that when calculating the normalized differences as displayed in Figs. 3 and 4, many systematic errors such as a nonuniform detection efficiency of the MCP (e.g., a "hole" in the detector or different angles of incidence relative to the MCP pores [13]) do not influence the final result, as they are normalized element by element. This is not the case 


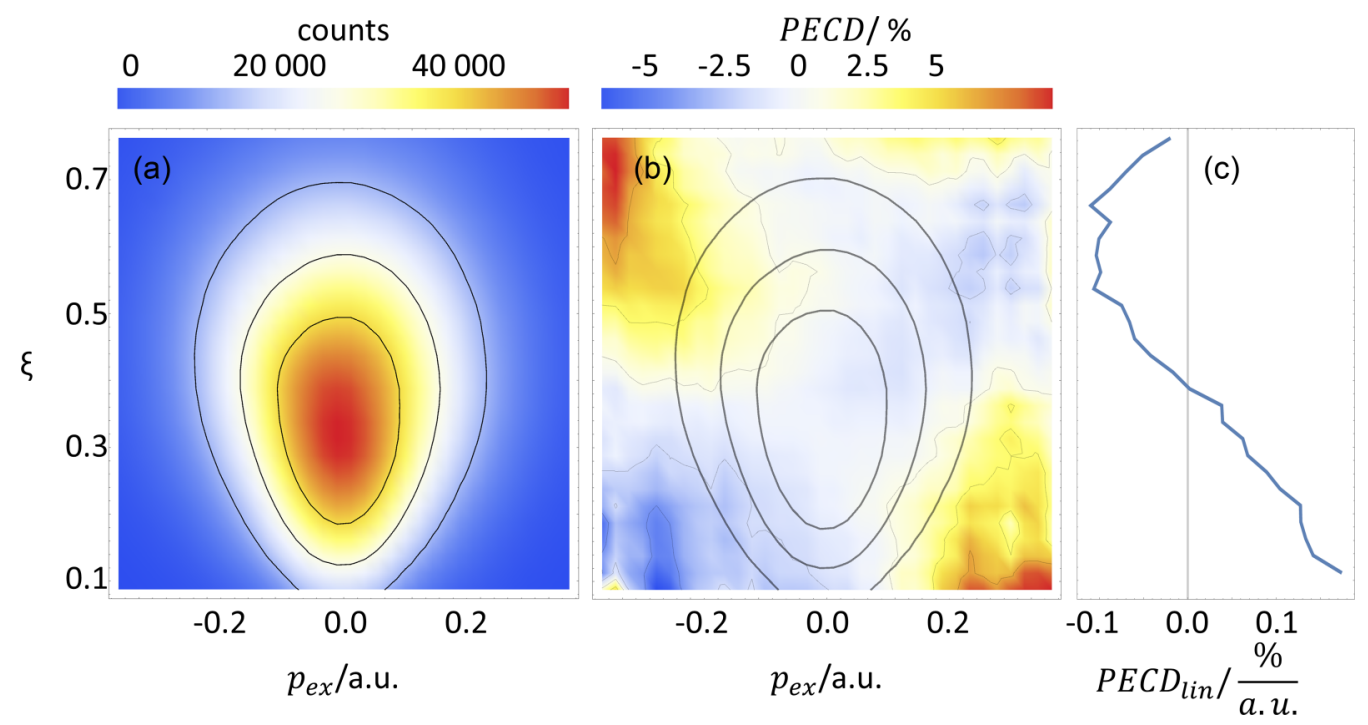

FIG. 3. PECD in the strong field regime with elliptically polarized light. (a) Distribution of the linear electron momenta in strong field ionization as a function of $p_{e x}$, the momentum collinear to the propagation direction of the light, and $\xi=\sqrt{p_{e y}^{2}+s^{2} p_{e z}^{2}}$, the component transversal to this direction for a selection in $\varphi$ as indicated in Fig. 1. (b) Normalized difference of (a) between the distribution of the $R$ and the $S$ enantiomer for LEP. The contour lines are the same as plotted in (a). (c) $\mathrm{PECD}_{\text {lin }}$ calculated as the linear fit from cuts in $\xi$.

for the Gaussian fits performed in Figs. 1 and 2. However, systematic errors are also less pronounced here, if the focus lies on the difference between the enantiomers. Presumably, this is the main reason for the systematic errors in Fig. 1(b) (especially at larger $\xi$ ) or Fig. 1(c), where the expected symmetry $\varphi_{\max , R}\left(p_{e x}\right)=\varphi_{\max , S}\left(-p_{e x}\right)$ is not perfectly satisfied, while the difference between both enantiomers generates a clear signal [colored areas in Fig. 1(c)].

\section{V. $\varphi$-DEPENDENT PHOTOELECTRON CIRCULAR DICHROISM}

In the next paragraphs, we connect this enantiospecific rotation of the momentum distribution to the more established quantity of PECD. In the strong field regime, the electron momentum distributions maximize in the polarization plane [Fig. 3(a)]. Hence, the forward/backward asymmetry quantified by PECD becomes visible as a small shift of the electron momenta distribution parallel to $p_{e x} . \operatorname{PECD}\left(\varphi, p_{e x}, \xi\right)$ is defined as the normalized difference of the number of ionization events between the $R$ and $S$ enantiomer $\left[N_{R}\left(\varphi, p_{e x}, \xi\right)\right.$ and $\left.N_{S}\left(\varphi, p_{e x}, \xi\right)\right]$ at a given $p_{e x}, \xi$, and $\varphi$ for a given helicity of the light.

$$
\operatorname{PECD}\left(\varphi, p_{e x}, \xi\right)=\frac{N_{R}\left(\varphi, p_{e x}, \xi\right)-N_{S}\left(\varphi, p_{e x}, \xi\right)}{N_{R}\left(\varphi, p_{e x}, \xi\right)+N_{S}\left(\varphi, p_{e x}, \xi\right)} .
$$

For circularly polarized light, PECD is independent of $\varphi$, and switching the enantiomer is equivalent to inverting the helicity of light, thus the definition of PECD as the normalized difference between ionization yields for the two helicities is equivalent to the definition for PECD as given in Eq. (1). This is not the case for elliptically polarized light and we use the definition (1) throughout this paper.

Figure 3(a) shows the electron momentum distribution in $p_{e x}$ and $\xi$ where $\varphi$ is integrated over the region indicated by the green shaded area in Fig. 1. Figure 3(b) shows the
PECD for the data displayed in (a), which reaches values of up to about $10 \%$ and displays a strong dependence on $\xi$. To characterize the forward/backward asymmetry of the PECD in $\varphi$ and $\xi$ coordinates, the slope of the linear fit of the $p_{e x}$ dependence of the PECD is used [Fig. 3(c)].

$$
\operatorname{PECD}_{\text {lin }}(\varphi, \xi)=\frac{\text { alinear fit }\left[\operatorname{PECD}\left(\varphi, p_{e x}, \xi\right)\right]}{\partial \text { pex }} .
$$

This parameter is similar to the forward/backward asymmetry of PECD known from the single- and multiphoton ionization experiments [18]. The main difference, however, is that here we do not integrate photoelectron momentum distribution over forward and backward hemispheres, which would result in a single asymmetry parameter, but define the forward/backward photoelectron asymmetry for every point in the polarization plane.

To connect PECD to angular streaking $\left(\varphi_{\max }\right)$ as discussed above, we examine the dependence of $\mathrm{PECD}_{\text {lin }}$ on the streaking angle $\varphi$ in Fig. 4. The contour lines in Fig. 4(a) correspond to the density of ionization events from Fig. 1 (LEP) in elliptical coordinates. The color coding shows the corresponding $\operatorname{PECD}_{\text {lin }}(\xi, \varphi)$. Lineouts of these data at 0.48 a.u. $<\xi<$ 0.58 a.u. around the maximum of the count rate are shown in Fig. 4(b). Both the contour lines for the counts and the $\operatorname{PECD}_{\text {lin }}(\xi, \varphi)$ show the twofold symmetry that is expected for elliptically polarized light.

Clearly, the angle of the maximum value of $\mathrm{PECD}_{\text {lin }}$ does not coincide with the angle where the maximum of the momentum distribution resides. Figure 4(c) shows the same dependences for a clockwise rotating laser field.

This finding has two important consequences. Firstly, the symmetries of PECD known for ionization by circularly polarized light do not hold anymore. For circularly polarized light the electron forward/backward asymmetry reverses symmetrically upon mirroring either the molecule or reversing the helicity of the light [19]. With elliptical light the symmetry 


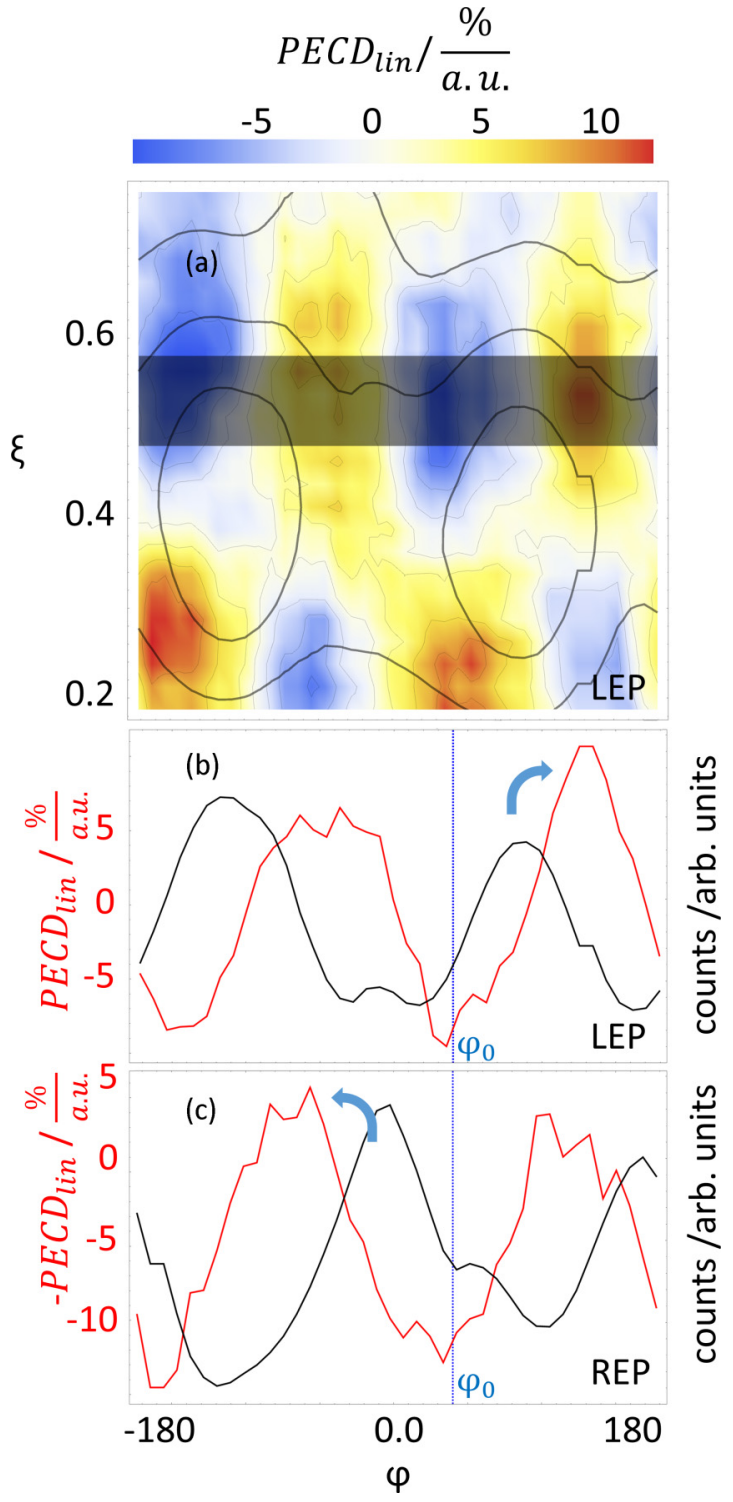

FIG. 4. PECD dependency on $\varphi$ for elliptically polarized light.

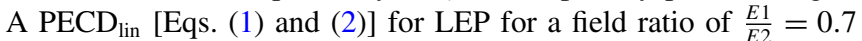
in elliptical coordinates. Thick contour lines indicate the count rates that enter the normalized difference. The $\mathrm{PECD}_{\text {lin }}$ shows a strong dependency on the angle $\varphi$. The $180^{\circ}$ symmetry of the $\mathrm{PECD}_{\text {lin }}$ is a consequence of the twofold symmetry of the elliptical light. Please note the sign change in $\mathrm{PECD}_{\text {lin }}$ at about $\xi=0.4$ a.u., which reflects the asymmetry inversion in $\varphi_{\max }$ in Fig. 1(b) also at about $\xi=0.4$ a.u. The second interesting feature is that the minimum and maximum values of $\mathrm{PECD}_{\text {lin }}$ do not appear at the same angle in the polarization plane as the maximum count rates. (b) Lineout of data in (a) for 0.48 a.u. $<\xi<0.58$ a.u. [gray shaded area (a)]. The dashed blue line at $\varphi_{0}$ indicates the position of the major axis of the laser polarization. (c) $-\mathrm{PECD}_{\text {lin }}$ for REP gating on 0.48 a.u. $<$ $\xi<0.58$ a.u. Note that while for LEP the maximum of the $\mathrm{PECD}_{\text {lin }}$ is shifted towards larger values in $\varphi$, for REP the maximum of the $-\mathrm{PECD}_{\text {lin }}$ is shifted towards smaller values in $\varphi$.

of PECD, upon inversion of the light helicity and not the enantiomer as in Eq. (1), is given by

$$
\operatorname{PECD}_{\operatorname{lin}}^{\mathrm{LEP}}\left(\varphi-\varphi_{0}\right)=-\operatorname{PECD}_{\operatorname{lin}}^{\mathrm{REP}}\left[-\left(\varphi-\varphi_{0}\right)\right] .
$$

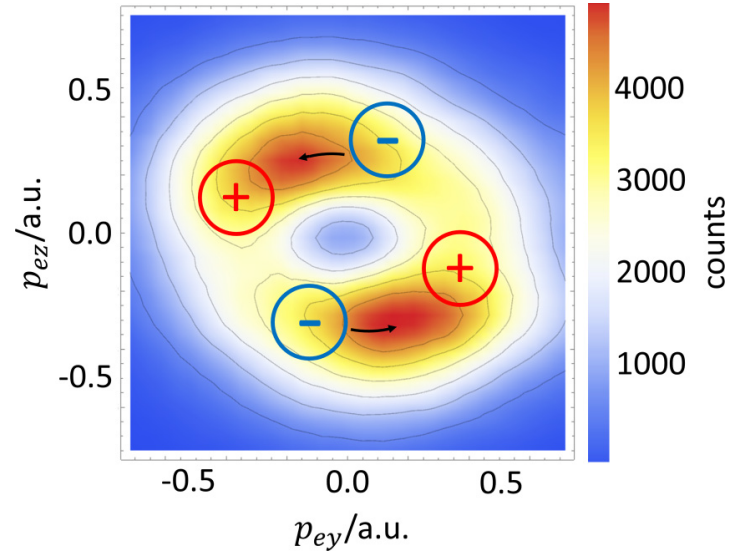

FIG. 5. Electron momentum distribution in the polarization plane $\left(p_{e y}-p_{e z}\right)$ for $p_{e x}=0.1 \pm 0.03$ a.u. measured with elliptically polarized light $\frac{E 1}{E 2}=0.7$. Areas in $\varphi$, in which the electron density is shifted in the direction of light propagation direction by the $\varphi$ dependent $\mathrm{PECD}_{\text {lin }}$ are marked with a red + , and where the electron density is shifted in opposite direction are marked with a blue -. Investigating the plane of polarization for a cut in light propagation direction ( $p_{e x}>0$ a.u.), the maxima of the electron distribution appear closer to the areas marked with the red + compared to a scenario in which $\mathrm{PECD}_{\text {lin }}$ does not depend on $\varphi$. This rotation of the maxima of the electron density is indicated with the black arrows.

Here REP and LEP refer to right- or left-hand sense of rotation of the electric field vector. $\varphi_{0}$ is the angle of the major axis of the electric field in the laboratory frame and $\varphi$ is the laboratory angle of the electron in the polarization plane. For circularly polarized light, $\varphi_{0}$ is arbitrary and Eq. (3) leads to the known symmetry that for PECD inverting the helicity is the same as exchanging the enantiomer. The second consequence is that PECD goes along with the enantiomer specific rotation of the count rate maxima shown in Fig. 1.

\section{CONNECTING THE $\varphi$-DEPENDENT PECD AND THE CHIRAL SIGNATURE ON THE STREAKING ANGLE $\varphi$}

Figure 5 shows the electron distribution in the plane of polarization $\left(p_{e y}-p_{e z}\right)$ for a cut in the light propagation direction with $p_{e x}=0.1 \pm 0.03$ a.u. As can be seen in Fig. 4(a), the maxima of $\left|\mathrm{PECD}_{\operatorname{lin}}\right|$ do not coincide with the maxima of the electron density. In Fig. 5 the location of these PECD $_{\text {lin }}$ extrema are shown by the red plus and blue minus signs, corresponding to positive and negative values of $\mathrm{PECD}_{\text {lin }}$.

Regions in the $p_{e y}-p_{e z}$ plane for momenta in the direction of light propagation $\left(p_{e x}>0\right)$ with a positive (negative) $\mathrm{PECD}_{\text {lin }}$ give rise to a slight increase (decrease) in the electron density. Consequently, the maximum of the electron density in the direction of the positive $\mathrm{PECD}_{\text {lin }}$ appears shifted/rotated (visualized by the black arrows). Due to the symmetry of the PECD, when the sign of the momentum component in the light propagation direction $\left(p_{e x}>0\right)$ changes, in the $p_{e y}-p_{e z}$ plane the increase and decrease of electron density interchanges. The resulting displacement/rotation of the electron density caused thereby takes place in the opposite direction. Hence, the properties of the enantiosensitive and 
forward/backward asymmetric rotation of the electron density as discussed in Fig. 1 are reproduced with this consideration with the $\varphi$-dependent PECD, whose extrema do not coincide with the maxima of the electron density. If the extrema of the $\varphi$-dependent PECD would coincide with the maxima of the electron density, the result would be an increase or decrease of the width of the electron density in $\varphi$, but not a rotation.

This chiral effect on angular streaking and the forward/backward shift encoded in PECD offer two different perspectives on the same phenomenon. Chiral molecules break the forward/backward symmetry of electron emission by elliptical light. The corresponding complex three-dimensional momentum distributions show rotated maxima as well as forward/backward asymmetries.

\section{CONCLUSION}

In this paper, we investigate the forward/backward asymmetric and enantiosensitive rotation of the photoelectron momentum distribution as a new chiral signal becoming accessible for elliptically polarized light upon strong field ionization.

If the rotational symmetry of circularly polarized light is broken by adding a linear component, it makes a difference whether the helicity of the ionizing light or the handedness of the molecule is inverted in a PECD measurement. This is a new aspect of PECD in elliptical light.
Elliptically polarized light leads to much richer chiral signals than circularly polarized light. This can be an asset if one aims for chiral recognition (compare Comby et al. [3]). It allows one to combine attosecond angular streaking with an enantiosensitive signal. Integrating over $p_{\text {ex }}$, the rotation angle of the count maximum can serve as a phase (or a time marker) for the ionization event. In these cases, the streaking angle is independent of the enantiomer. A difference of the rotation angle between the forward and backward values of $p_{e x}$ proves enantiomeric excess in the sample at the time of electron ejection. This can be applied, e.g., in pump-probe experiments where the observed streaking angle can serve as a subcycle time stamp [10] and the same signal can be used to test the chirality of the sample at the time of the pump step. The time evolution of the chirality can then be traced with a probe technique such as Coulomb explosion imaging [20].

\section{ACKNOWLEDGMENTS}

We are thankful for inspiring discussions with Kiyoshi Ueda. We acknowledge support from Deutsche Forschungsgemeinschaft via Sonderforschungsbereich 1319 (ELCH). K.F. and A.H. acknowledge support by the German National Merit Foundation. M.S. thanks the Adolf-Messer Foundation for financial support.

The authors declare no competing interests.
[1] R. Corradini, S. Sforza, T. Tedeschi, and R. Marchelli, Chirality 19, 269 (2007); D. Di Tommaso, M. Stener, G. Fronzoni, and P. Decleva, ChemPhysChem 7, 924 (2006); A. Ferré, C. Handschin, M. Dumergue, F. Burgy, A. Comby, D. Descamps, B. Fabre, G. A. Garcia, R. Géneaux, L. Merceron et al., Nat. Photonics 9, 93 (2015); L. Holmegaard, J. L. Hansen, L. Kalhøj, S. L. Kragh, H. Stapelfeldt, F. Filsinger, J. Küpper, G. Meijer, D. Dimitrovski, M. Abu-samha et al., Nat. Phys. 6, 428 (2010); P. Horsch, G. Urbasch, and K.-M. Weitzel, Chirality 24, 684 (2012); I. Powis, J. Phys. Chem. A 104, 878 (2000); Chirality 20, 961 (2008); Y. Zhang, J. R. Rouxel, J. Autschbach, N. Govind, and S. Mukamel, Chem. Sci. 8, 5969 (2017).

[2] G. A. Garcia, H. Dossmann, L. Nahon, S. Daly, and I. Powis, Phys. Chem. Chem. Phys. 16, 16214 (2014).

[3] A. Comby, E. Bloch, C. M. M. Bond, D. Descamps, J. Miles, S. Petit, S. Rozen, J. B. Greenwood, V. Blanchet, and Y. Mairesse, Nat. Commun. 9, 5212 (2018).

[4] R. E. Goetz, T. A. Isaev, B. Nikoobakht, R. Berger, and C. P. Koch, J. Chem. Phys. 146, 024306 (2017); I. Dreissigacker and M. Lein, Phys. Rev. A 89, 053406 (2014).

[5] A. Kastner, T. Ring, B. C. Krüger, G. B. Park, T. Schäfer, A. Senftleben, and T. Baumert, J. Chem. Phys. 147, 013926 (2017).

[6] P. Eckle, M. Smolarski, P. Schlup, J. Biegert, A. Staudte, M. Schöffler, H. G. Muller, R. Dörner, and U. Keller, Nat. Phys. 4, 565 (2008).

[7] P. Dietrich, F. Krausz, and P. B. Corkum, Opt. Lett. 25, 16 (2000).
[8] P. Eckle, A. N. Pfeiffer, C. Cirelli, A. Staudte, R. Dörner, H. G. Muller, M. Büttiker, and U. Keller, Science (NY) 322, 1525 (2008); U. S. Sainadh, H. Xu, X. Wang, A. Atia-Tul-Noor, W. C. Wallace, N. Douguet, A. Bray, I. Ivanov, K. Bartschat, A. Kheifets et al., Nature (London) 568, 75 (2019).

[9] M. S. Schöffler, X. Xie, P. Wustelt, M. Möller, S. Roither, D. Kartashov, A. M. Sayler, A. Baltuska, G. G. Paulus, and M. Kitzler, Phys. Rev. A 93, 063421 (2016); C. M. Maharjan, A. S. Alnaser, X. M. Tong, B. Ulrich, P. Ranitovic, S. Ghimire, Z. Chang, I. V. Litvinyuk, and C. L. Cocke, ibid. 72, 041403 (2005).

[10] J. Wu, M. Magrakvelidze, L. P. H. Schmidt, M. Kunitski, T. Pfeifer, M. Schöffler, M. Pitzer, M. Richter, S. Voss, H. Sann et al., Nat. Commun. 4, 2177 (2013).

[11] S. Eckart, K. Fehre, N. Eicke, A. Hartung, J. Rist, D. Trabert, N. Strenger, A. Pier, L. Ph. H. Schmidt, T. Jahnke et al., Phys. Rev. Lett. 121, 163202 (2018).

[12] R. Dörner, V. Mergel, O. Jagutzki, L. Spielberger, J. Ullrich, R. Moshammer, and H. Schmidt-Böcking, Phys. Rep. 330, 95 (2000).

[13] K. Fehre, D. Trojanowskaja, J. Gatzke, M. Kunitski, F. Trinter, S. Zeller, L. Ph. H. Schmidt, J. Stohner, R. Berger, A. Czasch et al., Rev. Sci. Instrum. 89, 045112 (2018).

[14] O. Jagutzki, A. Cerezo, A. Czasch, R. Dorner, M. Hattas, M. Huang, V. Mergel, U. Spillmann, K. Ullmann-Pfleger, T. Weber et al., IEEE Trans. Nucl. Sci. 49, 2477 (2002).

[15] C. Smeenk, J. Z. Salvail, L. Arissian, P. B. Corkum, C. T. Hebeisen, and A. Staudte, Opt. Express 19, 9336 (2011). 
[16] E. J. McAlduff and K. N. Houk, Can. J. Chem. 55, 318 (1977).

[17] K. Fehre, S. Eckart, M. Kunitski, C. Janke, D. Trabert, J. Rist, M. Weller, A. Hartung, L. Ph. H. Schmidt, T. Jahnke et al., J. Phys. Chem. A 123, 6491 (2019).

[18] L. Nahon and G. A. Garcia, J. Chem. Phys. 125, 114309 (2006); C. Lux, M. Wollenhaupt, C. Sarpe, and T. Baumert, ChemPhysChem 16, 115 (2015).

[19] C. Lux, M. Wollenhaupt, T. Bolze, Q. Liang, J. Köhler, C. Sarpe, and T. Baumert, Angew. Chem., Int. Ed. Engl. 51, 5001 (2012); C. S. Lehmann, N. B. Ram, I. Powis, and M. H. M.
Janssen, J. Chem. Phys. 139, 234307 (2013); G. A. Garcia, L. Nahon, M. Lebech, J.-C. Houver, D. Dowek, and I. Powis, ibid. 119, 8781 (2003).

[20] M. Pitzer, M. Kunitski, A. S. Johnson, T. Jahnke, H. Sann, F. Sturm, L. Ph. H. Schmidt, H. Schmidt-Böcking, R. Dörner, J. Stohner et al., Science (NY) 341, 1096 (2013); M. Pitzer, G. Kastirke, M. Kunitski, T. Jahnke, T. Bauer, C. Goihl, F. Trinter, C. Schober, K. Henrichs, J. Becht et al., ChemPhysChem 17, 2465 (2016); K. Fehre, S. Eckart, M. Kunitski, M. Pitzer, S. Zeller, C. Janke, D. Trabert, J. Rist, M. Weller, A. Hartung et al., Sci. Adv. 5, eaau7923 (2019). 